\title{
Joint Estimation of Carrier Frequency Offset and Channel Complex Gains for OFDM Systems in Fast Time-varying Vehicular Environments
}

\author{
Eric Simon, Hussein Hijazi, Laurent Ros, Marion Berbineau
}

\section{To cite this version:}

Eric Simon, Hussein Hijazi, Laurent Ros, Marion Berbineau. Joint Estimation of Carrier Frequency Offset and Channel Complex Gains for OFDM Systems in Fast Time-varying Vehicular Environments. ICC 2010 - IEEE International Conference on Communications, May 2010, Le Cap, South Africa. 5 p. hal-00539132

\section{HAL Id: hal-00539132 \\ https://hal.archives-ouvertes.fr/hal-00539132}

Submitted on 24 Nov 2010

HAL is a multi-disciplinary open access archive for the deposit and dissemination of scientific research documents, whether they are published or not. The documents may come from teaching and research institutions in France or abroad, or from public or private research centers.
L'archive ouverte pluridisciplinaire HAL, est destinée au dépôt et à la diffusion de documents scientifiques de niveau recherche, publiés ou non, émanant des établissements d'enseignement et de recherche français ou étrangers, des laboratoires publics ou privés. 


\title{
Joint Estimation of Carrier Frequency Offset and Channel Complex Gains for OFDM Systems in Fast Time-varying Vehicular Environments
}

\author{
Eric Pierre Simon ${ }^{1}$, Hussein Hijazi ${ }^{1}$, Laurent Ros $^{2}$, Marion Berbineau ${ }^{3}$ \\ ${ }^{1}$ IEMN lab, TELICE group, University of Lille, FRANCE \\ 2 GIPSA-lab, Department Image Signal, BP 46 - 38402 Saint Martin d'Hères, FRANCE \\ ${ }^{3}$ Universite Lille Nord de France, INRETS, LEOST, F-59650 Villeneuve dAscq \\ e-mail: eric.simon@univ-lille1.fr, hussein.hijazi@hotmail.fr, laurent.ros@gipsa-lab.inpg.fr, marion.berbineau@inrets.fr
}

\begin{abstract}
In this paper, the physical nature of the radiochannel is considered by using an $L$-path channel model to developing an algorithm for OFDM systems operating in fast timevarying vehicular environment. Assuming the Time of Arrival (TOA) is known, a novel iterative pilot-aided algorithm for joint estimation of multi-path Rayleigh channel complex gains and Carrier Frequency Offset (CFO) is introduced. Each complex gain time-variation, within one OFDM symbol, is approximated by a Basis Expansion Model (BEM) representation. An autoregressive $(A R)$ model is built for the parameters to be estimated (the AR model for the BEM coefficients is based on the Jakes process). The algorithm performs recursive estimation using Extended Kalman Filtering. Hence, the channel matrix is easily computed, and the data symbol is estimated with free intersub-carrier-interference (ICI) when the channel matrix is QRdecomposed. It is shown that only one iteration is sufficient to achieve the performance of the ideal case where knowledge of channel response and CFO is available.
\end{abstract}

\section{INTRODUCTION}

$\mathbf{O}$ RTHOGONAL frequency division multiplexing (OFDM) has become a standard technique for many wireless networks. However, it is well known that small carrier frequency offsets (CFOs) yield severe degradation in OFDM modulation since it produces inter-carrier interference (ICI) and attenuates the desired signal. These effects reduce the effective signal-to-noise ratio (SNR) in OFDM reception resulting in degraded system performance [1] [2]. CFOs are mainly caused by two different factors: Doppler effects and oscillator inaccuracies in the transmitter and receiver. Frequently, these two quantities are grouped together and modeled as a single frequency offset as in [3][4]. However, this model is not sufficiently accurate since separate offset parameter is needed for each propagation path given that the Doppler shift depends on the angle of arrival, which is peculiar to each path. Recently, it has been proposed to track directly the channel paths, which permits to take into account separate Doppler for each path [5][6]. Those works estimate the equivalent discrete-time channel taps ([6]) or the real path complex gains ([5]), which are both modeled by a basis expansion model (BEM). The BEM methods are Karhunen-Loeve BEM (KL-BEM), prolate spheroidal BEM (PS-BEM), complex exponential BEM (CE-BEM) and polynomial BEM (P-BEM).
However the CFO due to the mismatch between transmitter and receiver oscillators is not taken into account in those algorithms. In this paper, we propose a complete algorithm capable of estimating this CFO together with the time-variation of each channel path. To further improve the estimation accuracy, the algorithm uses decision feedback. Hence, the accuracy of the channel estimation, frequency offset estimation and symbol detection are enhanced simultaneously. Note also that since the pilots are used for both channel and frequency offset estimation, the pilot usage efficiency is greatly improved.

Generally, it is preferable to directly estimate the physical channel parameters [7] [5], instead of the equivalent discretetime channel taps [6]. Indeed, as the channel delay spread increases, the number of channel taps also increases, thus leading to a large number of BEM coefficients, and consequently more pilot symbols are needed. Estimating the physical propagation parameters means estimating multi-path TOAs and multi-path complex gains. Note that the TOAs can be safely assumed invariant (at least during a block of several OFDM symbols). In this work, they are assumed perfectly estimated. It should be noted that an initial, and generally accurate estimation of the number of paths and TOAs can be obtained by using the MDL (minimum description length) and ESPRIT (estimation of signal parameters by rotational invariance techniques) methods [7][8].

Generally, the CFO due to the oscillator mismatch is considered constant during the transmission. One reason for this is that oscillators drift with temperature, supply voltage, load, and other slowly changing environmental parameters. However, depending on communication duration, time-varying CFO can be significant in a real physical environment. Our algorithm is able to track the CFO in case of variation. Our algorithm is a recursive algorithm based on Extended Kalman Filtering (EKF) combined with QR-equalization for data detection.

This paper is organized as follows: Section II introduces the OFDM system and the BEM modeling. Section III describes the AR model for the BEM coefficients and the Extended Kalman Filter. Section IV covers the algorithm for joint channel estimation and data recovery. Section V presents the simulations results which validate our technique. Finally, our conclusions are presented in Section VI. 
The notations adopted are as follows: Upper (lower) bold face letters denote matrices (column vectors). $[\mathbf{x}]_{k}$ denotes the $k$ th element of the vector $\mathbf{x}$, and $[\mathbf{X}]_{k, m}$ denotes the $[k, m]$ th element of the matrix $\mathbf{X}$ (note that the indices begin from 0 ). $\mathbf{I}_{N}$ is a $N \times N$ identity matrix and $\mathbf{0}_{N}$ is a $N \times N$ matrix of zeros. $\operatorname{diag}\{\mathbf{x}\}$ is a diagonal matrix with $\mathbf{x}$ on its main diagonal and blkdiag $\{\mathbf{X}, \mathbf{Y}\}$ is a block diagonal matrix with the matrices $\mathbf{X}$ and $\mathbf{Y}$ on its main diagonal. The superscripts $(\cdot)^{T},(\cdot)^{*}$ and $(\cdot)^{H}$ stand respectively for transpose, conjugate and Hermitian operators. $\mathrm{E}[\cdot]$ is the expectation operation. $J_{0}(\cdot)$ is the zerothorder Bessel function of the first kind. $\nabla_{\mathbf{x}}$ represents the firstorder partial derivative operator i.e., $\nabla_{\mathbf{x}}=\left[\frac{\partial}{\partial x_{1}}, \ldots, \frac{\partial}{\partial x_{N}}\right]^{T}$.

\section{SYSTEM MODEL}

\section{A. OFDM System Model}

Consider an OFDM system with $N$ sub-carriers, and a cyclic prefix length $N_{g}$. The duration of an OFDM block is $T=N_{s} T_{s}$, where $T_{s}$ is the sampling time and $N_{s}=N+N_{g}$. Let $\mathbf{x}_{n}=\left[x_{n}\left[-\frac{N}{2}\right], x_{n}\left[-\frac{N}{2}+1\right], \ldots, x_{n}\left[\frac{N}{2}-1\right]\right]^{T}$ be the $n$th transmitted OFDM symbol and $\left\{x_{n}[b]\right\}$ are normalized symbols (i.e., $\mathrm{E}\left[x_{n}[b] x_{n}^{*}[b]\right]=1$ ). The frequency mismatch between the oscillators used in the radio transmitters and receivers causes a CFO $\Delta F$. The normalized CFO is denoted $\nu=\Delta F N T_{s}$. After transmission over a multi-path Rayleigh channel, the $n$th received OFDM symbol $\mathbf{y}_{n}$, where $\mathbf{y}_{n}=\left[y_{n}\left[-\frac{N}{2}\right], y_{n}\left[-\frac{N}{2}+1\right], \ldots, y_{n}\left[\frac{N}{2}-1\right]\right]^{T}$ is given by [9] [6]:

$$
\mathbf{y}_{n}=\mathbf{H}_{n} \mathbf{x}_{n}+\mathbf{w}_{n}
$$

where $\mathbf{w}_{n}=\left[w_{n}\left[-\frac{N}{2}\right], w_{n}\left[-\frac{N}{2}+1\right], \ldots, w_{n}\left[\frac{N}{2}-1\right]\right]^{T}$ is a white complex Gaussian noise vector of covariance matrix $\sigma^{2} \mathbf{I}_{N}$ and $\mathbf{H}_{n}$ is the $N \times N$ channel matrix. The elements of $\mathbf{H}_{n}$ can be written in terms of equivalent channel taps [10] $\left\{g_{l}^{(n)}\left(q T_{s}\right)=g_{l}\left(n T+q T_{s}\right)\right\}:$
$\quad\left[\mathbf{H}_{n}\right]_{k, m}=$
$\frac{1}{N} \sum_{l=0}^{L^{\prime}-1}\left[e^{-j 2 \pi\left(\frac{m}{N}-\frac{1}{2}\right) \cdot l} \sum_{q=0}^{N-1} e^{j 2 \pi \frac{\nu q}{N}} g_{l}^{(n)}\left(q T_{s}\right) e^{j 2 \pi \frac{m-k}{N} q}\right]$,

or in terms of physical channel parameters [8] (delays $\left\{\tau_{l}\right\}$ and complex gains $\left.\left\{\alpha_{l}^{(n)}\left(q T_{s}\right)=\alpha_{l}\left(n T+q T_{s}\right)\right\}\right)$, yielding:

$$
\begin{aligned}
& {\left[\mathbf{H}_{n}\right]_{k, m}=} \\
& \frac{1}{N} \sum_{l=0}^{L-1}\left[e^{-j 2 \pi\left(\frac{m}{N}-\frac{1}{2}\right) \tau_{l}} \sum_{q=0}^{N-1} e^{j 2 \pi \frac{\nu q}{N}} \alpha_{l}^{(n)}\left(q T_{s}\right) e^{j 2 \pi \frac{m-k}{N} q}\right] .
\end{aligned}
$$

$L^{\prime}<N_{g}$ and $L$ are respectively the number of channel taps and the number of paths. The delays are normalized by $T_{s}$ and not necessarily integers $\left(\tau_{l}<N_{g}\right)$. The $L$ elements of $\left\{\alpha_{l}^{(n)}\left(q T_{s}\right)\right\}$ are uncorrelated. However, the $L^{\prime}$ elements of $\left\{g_{l}^{(n)}\left(q T_{s}\right)\right\}$ are correlated, unless that the delays are multiple of $T_{s}$ as mostly assumed in the literature. They are wide-sense stationary (WSS), narrow-band zero-mean complex Gaussian processes of variances $\sigma_{g_{l}}{ }^{2}$ and $\sigma_{\alpha_{l}}{ }^{2}$, with the so-called Jakes' power spectrum of maximum Doppler frequency $f_{d}$ [11]. The average energy of the channel approach is normalized to one, i.e., $\sum_{l=0}^{L^{\prime}-1} \sigma_{g_{l}}{ }^{2}=1$ and $\sum_{l=0}^{L-1} \sigma_{\alpha_{l}}{ }^{2}=1$.

In the sequel, we will make the calculus based on the second approach (physical channel) and we can deduce the results of the first approach (channel taps) by replacing $L$ by $L^{\prime}$ and the set of delays $\left\{\tau_{l}\right\}$ by $\left\{l, l=0: L^{\prime}-1\right\}$.

\section{B. BEM Channel Model}

Since the number of samples to be estimated $L N_{s}$ is greater than the number of observation equations $N$, it is not efficient to estimate the time-variation of the complex gains, using directly the observation model in (1). Thus, we need to reduce the number of parameters to be estimated. In this section, our aim is to accurately model the timevariation of $\alpha_{l}^{(n)}\left(q T_{s}\right)$ by using a BEM. Collecting the samples of the $l$ th path within the $n$th OFDM symbol in a $N_{s} \times 1$ vector $\boldsymbol{\alpha}_{l}^{(n)}=\left[\alpha_{l}^{(n)}\left(-N_{g} T_{s}\right), \ldots, \alpha_{l}^{(n)}\left((N-1) T_{s}\right)\right]^{T}$, we can express $\boldsymbol{\alpha}_{l}^{(n)}$ as:

$$
\boldsymbol{\alpha}_{l}^{(n)}=\boldsymbol{\alpha}_{\mathbf{B E M}_{l}}^{(n)}+\boldsymbol{\xi}_{l}^{(n)}=\mathbf{Q} \mathbf{c}_{l}^{(n)}+\boldsymbol{\xi}_{l}^{(n)}
$$

where $\mathbf{Q}=\left[\mathbf{q}_{0}, \ldots, \mathbf{q}_{N_{c}-1}\right]$ is a $N_{s} \times N_{c}$ matrix that collects $N_{c}$ orthonormal basis function $\mathbf{q}_{d}$ as columns, $\mathbf{c}_{l}^{(n)}=$ $\left[c_{(0, l)}^{(n)}, \ldots, c_{\left(N_{c}-1, l\right)}^{(n)}\right]^{T}$ represents the $N_{c}$ BEM coefficients for the $l$ th complex gain of the $n$th OFDM symbol, and $\boldsymbol{\xi}_{l}^{(n)}$ represents the corresponding BEM modeling error, which is assumed to be minimized in the MSE sense [12] [13]. Under this criterion, the optimal BEM coefficients and the corresponding model error are given by:

$$
\begin{aligned}
& \mathbf{c}_{l}^{(n)}=\left(\mathbf{Q}^{H} \mathbf{Q}\right)^{-1} \mathbf{Q}^{H} \boldsymbol{\alpha}_{l}^{(n)} \\
& \boldsymbol{\xi}_{l}^{(n)}=\left(\mathbf{I}_{N_{s}}-\mathbf{S}\right) \boldsymbol{\alpha}_{l}^{(n)}
\end{aligned}
$$

where $\mathbf{S}=\mathbf{Q}\left(\mathbf{Q}^{H} \mathbf{Q}\right)^{-1} \mathbf{Q}^{H}$ is a $N_{s} \times N_{s}$ matrix. It provides the MMSE approximation for all BEM containing $N_{c}$ coefficients, given by:

$$
\begin{aligned}
& \operatorname{MMSE}_{l}=\frac{1}{N_{s}} \mathrm{E}\left[\boldsymbol{\xi}_{l}^{(n)} \boldsymbol{\xi}_{l}^{(n)}{ }^{H}\right] \\
& =\frac{1}{N_{s}} \operatorname{Tr}\left(\left(\mathbf{I}_{N_{s}}-\mathbf{S}\right) \mathbf{R}_{\boldsymbol{\alpha}_{l}}^{(0)}\left(\mathbf{I}_{N_{s}}-\mathbf{S}^{H}\right)\right)
\end{aligned}
$$

where $\mathbf{R}_{\boldsymbol{\alpha}_{l}}^{(s)}=\mathrm{E}\left[\boldsymbol{\alpha}_{l}^{(n)} \boldsymbol{\alpha}_{l}^{(n-s)^{H}}\right]$ is the $N_{s} \times N_{s}$ correlation matrix of $\boldsymbol{\alpha}_{l}^{(n)}$ with elements given by:

$$
\left[\mathbf{R}_{\boldsymbol{\alpha}_{l}}^{(s)}\right]_{k, m}=\sigma_{\alpha_{l}}^{2} J_{0}\left(2 \pi f_{d} T_{s}\left(k-m+s N_{s}\right)\right)
$$

Various traditional BEM designs have been reported to model the channels time-variations, e.g., the CE-BEM $[\mathbf{Q}]_{k, m}=e^{j 2 \pi\left(\frac{k-N g}{N_{s}}\right)\left(m-\frac{N_{c}-1}{2}\right)}[14]$, the GCE-BEM $[\mathbf{Q}]_{k, m}=$ $e^{j 2 \pi\left(\frac{k-N g}{a v}\right)\left(m-\frac{N_{c}-1}{2}\right)}$ with $1<a \leq \frac{N_{c}-1}{2 f_{d} T}$ [12], the P-BEM $[\mathbf{Q}]_{k, m}=(k-N g)^{m}$ [13] and the DKL-BEM which employs 
basis sequences that corresponds to the most significant eigenvectors of the autocorrelation matrix $\mathbf{R}_{\alpha_{l}}^{(0)}$ [15]. From now on, we can describe the OFDM system model derived previously in terms of the BEM. Substituting (4) in (1) and neglecting the BEM model error, we obtain after some algebra:

$$
\mathbf{y}_{n}=\mathcal{K}_{n}(\nu) \cdot \mathbf{c}_{n}+\mathbf{w}_{n}
$$

where the $L N_{c} \times 1$ vector $\mathbf{c}_{n}$ and the $N \times L N_{c}$ matrix $\mathcal{K}_{n}$ are given by:

$$
\begin{aligned}
\mathbf{c}_{n}= & {\left[\mathbf{c}_{0}^{(n)^{T}}, \ldots, \mathbf{c}_{L-1}^{(n)^{T}}\right]^{T} } \\
\mathcal{K}_{n}(\nu)= & \frac{1}{N}\left[\mathbf{Z}_{0}^{(n)}(\nu), \ldots, \mathbf{Z}_{L-1}^{(n)}(\nu)\right] \\
\mathbf{Z}_{l}^{(n)}(\nu)= & {\left[\mathbf{M}_{0}(\nu) \operatorname{diag}\left\{\mathbf{x}_{n}\right\} \mathbf{f}_{l}, \ldots,\right.} \\
& \left.\quad \mathbf{M}_{N_{c}-1}(\nu) \operatorname{diag}\left\{\mathbf{x}_{n}\right\} \mathbf{f}_{l}\right]
\end{aligned}
$$

where vector $\mathbf{f}_{l}$ is the $l$ th column of the $N \times L$ Fourier matrix $\mathbf{F}$ and $\mathbf{M}_{d}(\nu)$ is a $N \times N$ matrix given by:

$$
\begin{aligned}
{[\mathbf{F}]_{k, l} } & =e^{-j 2 \pi\left(\frac{k}{N}-\frac{1}{2}\right) \tau_{l}} \\
{\left[\mathbf{M}_{d}(\nu)\right]_{k, m} } & =\sum_{q=0}^{N-1} e^{j 2 \pi \frac{\nu q}{N}}[\mathbf{Q}]_{q+N_{g}, d} e^{j 2 \pi \frac{m-k}{N} q}
\end{aligned}
$$

Moreover, the channel matrix can be easily computed by using the BEM coefficients [9]:

$$
\mathbf{H}_{n}=\sum_{d=0}^{N_{c}-1} \mathbf{M}_{d}(\nu) \operatorname{diag}\left\{\mathbf{F} \boldsymbol{\chi}_{d}^{(n)}\right\}
$$

where $\chi_{d}^{(n)}=\left[c_{(d, 0)}^{(n)}, \ldots, c_{(d, L-1)}^{(n)}\right]^{T}$.

\section{AR Model And Extended Kalman Filter}

\section{A. The AR Model for $\boldsymbol{c}_{n}$}

The optimal BEM coefficients $\mathbf{c}_{l}^{(n)}$ are correlated complex Gaussian variables with zero-means and correlation matrix given by:

$$
\begin{aligned}
\mathbf{R}_{\mathbf{c}_{l}}^{(s)} & =\mathrm{E}\left[\mathbf{c}_{l}^{(n)} \mathbf{c}_{l}^{(n-s)^{H}}\right] \\
& =\left(\mathbf{Q}^{H} \mathbf{Q}\right)^{-1} \mathbf{Q}^{H} \mathbf{R}_{\boldsymbol{\alpha}_{l}}^{(s)} \mathbf{Q}\left(\mathbf{Q}^{H} \mathbf{Q}\right)^{-1}
\end{aligned}
$$

Hence, the dynamics of $\mathbf{c}_{l}^{(n)}$ can be well modeled by an autoregressive (AR) process [16] [17] [8] . A complex AR process of order $p$ can be generated as:

$$
\mathbf{c}_{l}^{(n)}=\sum_{i=1}^{p} \mathbf{A}^{(i)} \mathbf{c}_{l}^{(n-i)}+\mathbf{u}_{l}^{(n)}
$$

where $\mathbf{A}^{(1)}, \ldots, \mathbf{A}^{(p)}$ are $N_{c} \times N_{c}$ matrices and $\mathbf{u}_{l}^{(n)}$ is a $N_{c} \times 1$ complex Gaussian vector with covariance matrix $\mathbf{U}_{l}$. From [9], it is sufficient to choose $p=1$ to correctly model the coefficients. The matrices $\mathbf{A}^{(1)}=\mathbf{A}$ and $\mathbf{U}_{l}$ are the AR model parameters obtained by solving the set of Yule-Walker equations defined as:

$$
\begin{aligned}
\mathbf{A} & =\mathbf{R}_{\mathbf{c}_{l}}^{(1)}\left(\mathbf{R}_{\mathbf{c}_{l}}^{(0)}\right)^{-1} \\
\mathbf{U}_{l} & =\mathbf{R}_{\mathbf{c}_{l}}^{(0)}+\mathbf{A} \mathbf{R}_{\mathbf{c}_{l}}^{(-1)}
\end{aligned}
$$

Using (18), we obtain the AR model of order 1 for $\mathbf{c}_{n}$ :

$$
\mathbf{c}_{n}=\mathcal{A}_{\mathbf{c}} \cdot \mathbf{c}_{n-1}+\mathbf{u}_{\mathbf{c} n}
$$

where $\mathcal{A}_{\mathbf{c}}=\operatorname{blkdiag}\{\mathbf{A}, \ldots, \mathbf{A}\}$ is a $L N_{c} \times L N_{c}$ matrix and $\mathbf{u}_{\mathbf{c} n}=\left[\mathbf{u}_{0}^{(n)^{T}}, \ldots, \mathbf{u}_{L_{-}}^{(n)^{T}}\right]^{T}$ is a $L N_{c} \times 1$ zero-mean complex Gaussian vector with covariance matrix $\mathbf{U}_{\mathbf{c}}=$ blkdiag $\left\{\mathbf{U}_{0}, \ldots, \mathbf{U}_{L-1}\right\}$.

\section{B. The AR Model for $\nu_{n}$}

Let us write the order $1 \mathrm{AR}$ model for $\nu_{n}$ as follows:

$$
\nu_{n}=a \cdot \nu_{n-1}+u_{\nu_{n}}
$$

Since the CFO can be assumed as constant during the observation interval, $a$ is considered to be close to $1, a=0.99$. The state noise parameter $u_{\nu_{n}}$ is assumed to be zero-mean complex Gaussian with variance $\sigma_{\nu}^{2}$.

\section{State equation}

Now, let us write the state-variable model. The state vector at time instance $n$ consists of the BEM coefficients $\mathbf{c}_{n}$ and the vector of CFO $\nu_{n}$ :

$$
\boldsymbol{\mu}_{n}=\left[\mathbf{c}_{n}^{T}, \nu_{n}^{T}\right]^{T}
$$

There are $L N_{c}$ BEM coefficients and $1 \mathrm{CFO}$ values in the state vector of dimension $L N_{c}+1 \times 1$. Then the linear state equation may be written as follows:

$$
\boldsymbol{\mu}_{n}=\mathcal{A} \cdot \boldsymbol{\mu}_{n-1}+\mathbf{u}_{n}
$$

where the state transition matrix is defined as follows:

$$
\mathcal{A}=\operatorname{blkdiag}\left\{\mathcal{A}_{\mathfrak{c}}, a\right\}
$$

The $L N_{c}+1 \times 1$ noise vector is such that $\mathbf{u}_{n}=\left[\mathbf{u}_{\mathbf{c} n}^{T}, u_{\nu_{n}}\right]^{T}$ with covariance matrix $\mathbf{U}=\operatorname{blkdiag}\left\{\mathbf{U}_{\mathbf{c}}, \sigma_{\nu}^{2}\right\}$.

\section{Extended Kalman Filter (EKF)}

The measurement equation (10) can be reformulated as:

$$
\mathbf{y}_{n}=\mathbf{g}\left(\boldsymbol{\mu}_{n}\right)+\mathbf{w}_{n}
$$

where the nonlinear function $\mathbf{g}$ of the state vector $\boldsymbol{\mu}_{n}$ is defined as $\mathbf{g}\left(\boldsymbol{\mu}_{n}\right)=\mathcal{K}_{n}(\nu) \cdot \mathbf{c}_{n}$. Nonlinearity of the measurement equation (26) is caused by CFO. The BEM coefficients are still linearly related to observations. Since the measurement equation is nonlinear, we use the Extended Kalman filter to adaptively track $\boldsymbol{\mu}_{n}$. Let $\hat{\boldsymbol{\mu}}_{(n \mid n-1)}$ be our a priori state estimate at step $n$ given knowledge of the process prior to step $n, \hat{\boldsymbol{\mu}}_{(n \mid n)}$ be our a posteriori state estimate at step $n$ given measurement $\mathbf{y}_{n}$ and, $\mathbf{P}_{(n \mid n-1)}$ and $\mathbf{P}_{(n \mid n)}$ are the a priori and the a posteriori error estimate covariance matrix of size $L N_{c}+1 \times L N_{c}+1$, respectively. We initialize the EKF with $\hat{\boldsymbol{\mu}}_{(0 \mid 0)}=\mathbf{0}_{L N_{c}+1,1}$ and $\mathbf{P}_{(0 \mid 0)}$ given by:

$$
\begin{aligned}
\mathbf{P}_{(0 \mid 0)} & =\operatorname{blkdiag}\left\{\mathbf{R}_{\mathbf{c}}^{(0)}, b\right\} \\
\mathbf{R}_{\mathbf{c}}^{(s)} & =\operatorname{blkdiag}\left\{\mathbf{R}_{\mathbf{c}_{0}}^{(s)}, \ldots, \mathbf{R}_{\mathbf{c}_{L-1}}^{(s)}\right\}
\end{aligned}
$$


where $\mathbf{R}_{\mathbf{c}_{l}}^{(s)}$ is the correlation matrix of $\mathbf{c}_{l}^{(n)}$ defined in (17). To derive the EKF equations, we need to compute the Jacobian matrix $\mathbf{G}_{n}$ of $\mathbf{g}\left(\boldsymbol{\mu}_{n}\right)$ with respect to $\boldsymbol{\mu}_{n}$ and evaluated at $\hat{\boldsymbol{\mu}}_{(n \mid n-1)}$ :

$$
\begin{aligned}
& \mathbf{G}_{n}=\left.\nabla_{\boldsymbol{\mu}_{n}}^{T} \mathbf{g}\left(\boldsymbol{\mu}_{n}\right)\right|_{\boldsymbol{\mu}_{n}=\hat{\boldsymbol{\mu}}_{(n \mid n-1)}}= \\
& {\left[\left.\nabla_{\mathbf{c}_{n}}^{T} \mathbf{g}\left(\boldsymbol{\mu}_{n}\right)\right|_{\boldsymbol{\mu}_{n}=\hat{\boldsymbol{\mu}}_{(n \mid n-1)}},\left.\nabla_{\nu_{n}}^{T} \mathbf{g}\left(\boldsymbol{\mu}_{n}\right)\right|_{\boldsymbol{\mu}_{n}=\hat{\boldsymbol{\mu}}_{(n \mid n-1)}}\right]}
\end{aligned}
$$

After computation, we find:

$$
\mathbf{G}_{n}=\left[\mathcal{K}_{n}\left(\hat{\nu}_{(n \mid n-1)}\right), \mathcal{K}_{n}^{\prime}\left(\hat{\nu}_{(n \mid n-1)}\right) \hat{\mathbf{c}}_{(n \mid n-1)}\right]
$$

where

$$
\begin{aligned}
\mathcal{K}_{n}^{\prime}(\nu) & =\frac{1}{N}\left[\mathbf{Z}_{0}^{\prime(n)}(\nu), \ldots, \mathbf{Z}_{L-1}^{(n)}(\nu)\right] \\
\mathbf{Z}_{l}^{\prime(n)}(\nu)= & {\left[\mathbf{M}_{0}^{\prime}(\nu) \operatorname{diag}\left\{\mathbf{x}_{n}\right\} \mathbf{f}_{l}, \ldots,\right.} \\
& \left.\mathbf{M}_{N_{c}-1}^{\prime}(\nu) \operatorname{diag}\left\{\mathbf{x}_{n}\right\} \mathbf{f}_{l}\right]
\end{aligned}
$$

The $N \times N$ matrix $\mathbf{M}_{d}^{\prime}(\nu)$ is given by:

$$
\left[\mathbf{M}_{d}^{\prime}(\nu)\right]_{k, m}=\sum_{q=0}^{N-1} j 2 \pi \frac{q}{N} e^{j 2 \pi \frac{\nu q}{N}}[\mathbf{Q}]_{q+N_{g}, d} e^{j 2 \pi \frac{m-k}{N} q}
$$

The EKF is a recursive algorithm composed of two stages: Time Update Equations and Measurement Update Equations. These two stages are defined as:

\section{Time Update Equations:}

$$
\begin{aligned}
& \hat{\boldsymbol{\mu}}_{(n \mid n-1)}=\mathcal{A} \hat{\boldsymbol{\mu}}_{(n-1 \mid n-1)} \\
& \mathbf{P}_{(n \mid n-1)}=\mathcal{A} \mathbf{P}_{(n-1 \mid n-1)} \mathcal{A}^{H}+\mathbf{U}
\end{aligned}
$$

\section{Measurement Update Equations:}

$$
\begin{aligned}
\mathbf{K}_{n} & =\mathbf{P}_{(n \mid n-1)} \mathbf{G}_{n}^{H}\left(\mathbf{G}_{n} \mathbf{P}_{(n \mid n-1)} \mathbf{G}_{n}^{H}+\sigma^{2} \mathbf{I}_{N}\right)^{-1} \\
\hat{\boldsymbol{\mu}}_{(n \mid n)} & =\hat{\boldsymbol{\mu}}_{(n \mid n-1)}+\mathbf{K}_{n}\left(\mathbf{y}_{n}-\mathbf{g}\left(\hat{\boldsymbol{\mu}}_{(n \mid n-1)}\right)\right) \\
\mathbf{P}_{(n \mid n)} & =\mathbf{P}_{(n \mid n-1)}-\mathbf{K}_{n} \mathbf{G}_{n} \mathbf{P}_{(n \mid n-1)}
\end{aligned}
$$

where $\mathbf{K}_{n}$ is the Kalman gain. The Time Update Equations are responsible for projecting forward (in time) the current state and error covariance estimates to obtain the a priori estimates for the next time step. The Measurement Update Equations are responsible for the feedback, i.e., for incorporating a new measurement into the a priori estimate to obtain an improved a posteriori estimate. The Time Update Equations can also be thought of a predictor equations, while the Measurement Update Equations can be thought of a corrector equations.

\section{JoInt DATA DETECTION AND EKF}

In the iterative algorithm for joint data detection, channel and CFO extended Kalman estimation, the $N_{p}$ pilots subcarriers are evently inserted into the $\mathrm{N}$ subcarriers at the positions $\mathcal{P}=\left\{p_{r} \mid p_{r}=(r-1) L_{f}+1, r=1, \ldots, N_{p}\right\}$, where $L_{f}$ is the distance between two adjacent pilots. We use the the QR-equalizer [8] [18] for the data detection. The QRequalizer allows us to estimate the data symbol with free ICI by performing a so-called QR-decomposition. The algorithm proceeds as follows:

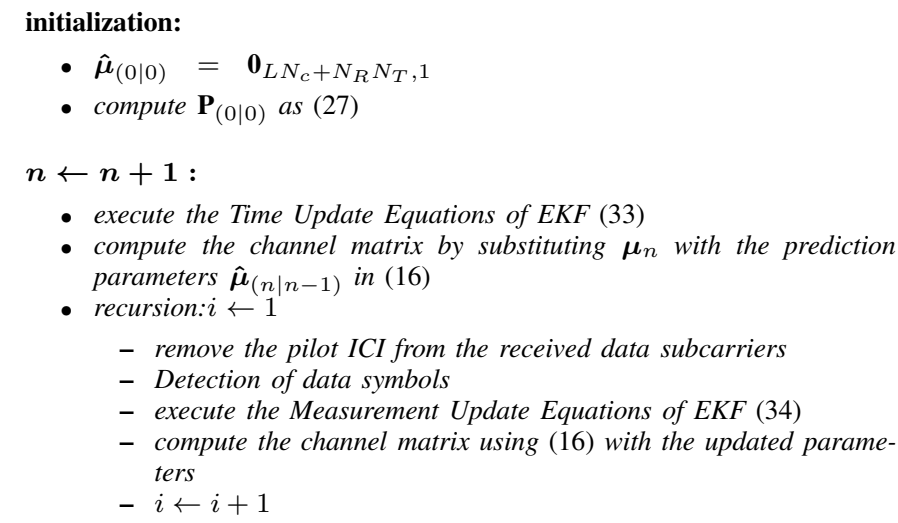

where $i$ represents the iteration number.

\section{Simulation}

In this section, the performance of our recursive algorithm is evaluated in terms of MSE for the channel and CFO estimation and BER for data detection. The normalized channel model is GSM Rayleigh model with $L=6$ paths and maximum delay $\tau_{\max }=10 T_{s}$ (see table I) [19][20][9]. A 4QAM-OFDM system with normalized symbols, $N=128$ subcarriers, $N_{g}=\frac{N}{8}$ subcarriers, $N_{p}=32$ pilots (i.e., $\left.L_{f}=4\right)$ and $\frac{1}{T_{s}}=2 M H z$ is used (note that $(S N R) d B=\left(\frac{E_{b}}{N_{0}}\right) d B+3 d B$ ). These parameters are selected in order to be in concordance with the standard Wimax IEEE802.16e. The MSE and the BER are evaluated under a rapid time-varying channel such as $f_{d} T=0.1$ corresponding to a vehicle speed $V_{m}=600 \mathrm{~km} / \mathrm{h}$ for $f_{c}=2.5 G H z$. We choose a GCE-BEM [12] and $N_{c}=3$ coefficients to model the path complex gain of the channel. Most advanced technologies have oscillator frequency tolerance less than $1 \mathrm{ppm}$ (i.e. $\nu=0.16$ in normalized units). For the simulation, we choose $\nu=0.1$.

Fig. 1 shows the MSE as a function of SNR. The MSE is simulated for three iterations and is shown for the CFO on the one hand and for the multi-path complex gain on the other hand. For reference, the MSEs obtained in Data Aided (DA) mode have been plotted. As expected, the MSEs obtained in Data Aided mode are lower than the MSEs obtained with just the pilots, especialy at low SNR where the detection errors are the most important. At high SNR, the MSEs tend to the MSEs in DA mode. It is also observed that after $20 \mathrm{~dB}$, there is no improvement any more. This is due to the fact that beyond 20 $\mathrm{dB}$, the matrix to be inversed to compute the Kalman Gain in Eq. (34) becomes badly scaled. This issue can be attenuated by adding some noise on the main diagonal of this matrix.

Fig. 2 gives the BER performance of our proposed iterative algorithm. For reference, we also plotted performance obtained with perfect knowledge of channel response and CFO. It is shown that after just one iteration, our joint estimator can achieve the same Bit Error Rate (BER) as that of an ideal reference receiver with perfect knowledge of channel response and carrier frequency offset. This shows that our proposed iterative joint channel and frequency offset estimator is effective. Again, it is observed a degradation in the performance after $20 \mathrm{~dB}$. However, most of the typical modern systems operate with a SNR less than $20 \mathrm{~dB}$. 


\begin{tabular}{|c|c|c|}
\hline Path number & Average Power $(\mathrm{dB})$ & Delay $\left(T_{s}\right)$ \\
\hline \hline 0 & -7.219 & 0 \\
\hline 1 & -4.219 & 0.4 \\
\hline 2 & -6.219 & 1 \\
\hline 3 & -10.219 & 3.2 \\
\hline 4 & -12.219 & 4.6 \\
\hline 5 & -14.219 & 10 \\
\hline
\end{tabular}

TABLE I

RAYLEIGH CHANNEL PARAMETERS

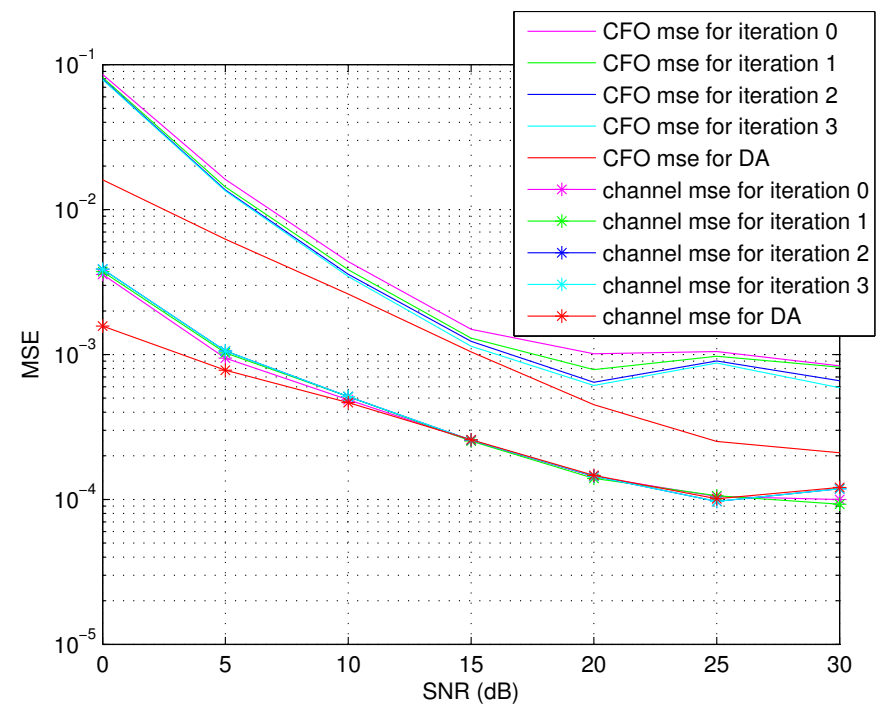

Fig. 1. Mean Square Error (MSE) as a function of SNR for $f_{d} T=0.1$

\section{CONCLUSION}

A new iterative algorithm which jointly estimates multipath complex gain and CFO has been presented. The algorithm is based on a parametric channel model. Extended Kalman filtering is used for parameter estimation and the data recovery is carried out by means of a QR-equalizer. Simulation results show that by estimating and removing the ICI at each iteration, the BER is greatly improved, especially after the first iteration. For a SNR $<20 \mathrm{~dB}$ (typical modern systems), our algorithm needs only one iteration to achieve the same performance than the ideal case where knowledge of the channel response and $\mathrm{CFO}$ is available. However, for a SNR $>20 \mathrm{~dB}$, performance is degraded compared to the ideal case.

\section{ACKOWLEDGEMENT}

This work has been carried out in the framework of the CISIT (Campus International sur la Sécurité et 1 Intermodalité des Transports) project and funded by the French Ministry of Research, the Region Nord Pas de Calais and the European Commission (FEDER funds)

\section{REFERENCES}

[1] T. Pollet, M. V. Bladel, and M. Moeneclaey, "BER Sensitivity of OFDM Systems to Carrier Frequency Offset and Wiener Phase Noise," IEEE Trans. Commun., vol. 43, no. 2/3/4, pp. 191 - 193, February/March/April 1995.

[2] H. Steendam and M. Moeneclaey, "Sensitivity of Orthogonal FrequencyDivision Multiplexed Systems to Carrier and Clock Synchronisation Errors," Signal Processing, vol. 80, pp. 1217-1229, 2000.

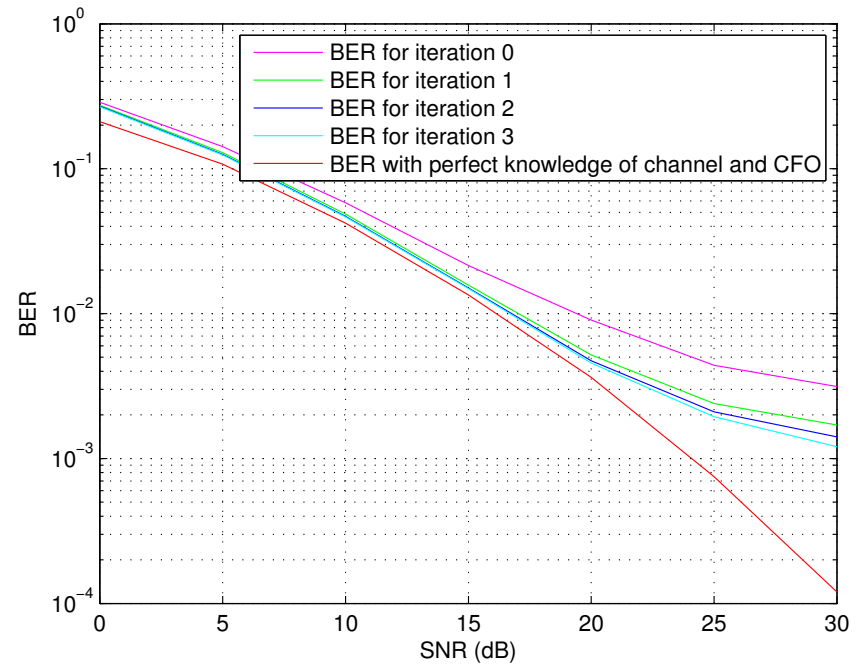

Fig. 2. Bit Error Rate (BER) as a function of SNR for $f_{d} T=0.1$

[3] P. H. Moose, "A technique for orthogonal frequency division multiplexing frequency offset correction," IEEE Trans. Commun, vol. 42, pp. 2908-2914, 1994.

[4] T. Roman, M. Enescu, and V. Koivunen, "Joint Time-Domain Tracking of Channel and Frequency Offsets for MIMO OFDM Systems," Wireless Personal Communications, vol. 31, pp. 181-200, 2004.

[5] H. Hijazi and L. Ros, "Time-varying channel complex gains estimation and ICI suppression in OFDM systems," in IEEE GLOBAL COMMUNICATIONS Conf., 2007.

[6] Z. Tang, R. C. Cannizzaro, G. Leus, and P. Banelli, "Pilot-assisted timevarying channel estimation for OFDM systems," IEEE Trans. Signal Process., vol. 55, pp. 2226-2238, 2007.

[7] B. Yang, K. B. Letaief, R. S. Cheng, and Z. Cao, "Channel estimation for OFDM transmisson in mutipath fading channels based on parametric channel modeling," IEEE Trans. Commun., vol. 49, pp. 467-479, 2001.

[8] H. Hijazi and L. Ros, "Joint Data QR-Detection and Kalman Estimation for OFDM Time-varying Rayleigh Channel Complex Gains," IEEE Trans. Comm., to be appeared in 2010.

[9] — , "Polynomial estimation of time-varying multi-path gains with intercarrier interference mitigation in OFDM systems," IEEE Trans. Vehic. Techno., vol. 57, 2008.

[10] J.-G. Kim and J.-T. Lim, "MAP-Based Channel Estimation for MIMOOFDM Over Fast Rayleigh Fading Channels," IEEE Trans. Vehic. Techno., vol. 57, 2008.

[11] W. C. Jakes, Microwave Mobile Communications. IEEE Press, 1983.

[12] G. Leus, "On the Estimation of Rapidly Time-Varying Channels," in Euro. Signal Process. Conf. (EUSIPCO), 2004.

[13] H. Hijazi and L. Ros, "Analytical Analysis of Bayesian Cramer-Rao Bound for Dynamical Rayleigh Channel Complex Gains Estimation in OFDM System," IEEE Trans. Signal Process., vol. 57, 2009.

[14] K. D. Teo and S. Ohno, "Optimal MMSE Finite Parameter Model for Doubly-selective Channels," in Proc. IEEE GLOBAL COMMUNICATIONS Conf., 2005.

[15] A. R. Kannu and P. Schniter, "MSE-optimal Training for Linear Timevarying Channels," in IEEE ICASSP Conf., 2005.

[16] K. E. Baddour and N. C. Beaulieu, "Autoregressive modeling for fading channel simulation," IEEE Trans. Wireless Commun., vol. 4, pp. 1650$1662,2005$.

[17] B. Anderson and J. B. Moore, Optimal filtering. Prentice-Hall, 1979.

[18] H. Hijazi and L. Ros, "OFDM High Speed Channel Complex Gains Estimation Using Kalman Filter and QR-Detector," in IEEE ISWCS Conf., 2008.

[19] Y. Zhao and A. Huang, "A novel Channel estimation method for OFDM Mobile Communications Systems based on pilot signals and transform domain processing," in IEEE 47th Vehicular Techno. Conf., May 1997, pp. 2089-2093.

[20] H. Hijazi and L. Ros, "Rayleigh Time-varying Channel Complex Gains Estimation and ICI Cancellation in OFDM Systems," European Transactions on Telecommunications, 2009. 